\title{
Les dirigeants français du CAC 40 : entre élitisme scolaire et passage par l’État
}

\author{
François-Xavier Dudouet ${ }^{1}$ et Hervé JoLY ${ }^{2}$
}

Cet article analyse les formations supérieures des dirigeants français des entreprises du CAC 40 à la fin 2007. Il existe toujours une forte prédominance des anciens élèves des "très grandes écoles " (HEC rejoignant Polytechnique et I'ENA), avec un avantage maintenu pour les membres des grands corps de l'État.

Les recherches sur le recrutement des élites économiques françaises, qui avaient connu un vif engouement à la fin des années 1970, sont par la suite quelque peu tombées en désuétude à l'exception notable des travaux de Michel Bauer et Bénédicte Bertin-Mourot pour les années 1980-1990³ . Au-delà de leurs divergences d'approches, ces enquêtes s'entendaient pour reconnaître un fort tropisme du système des grandes écoles et du passage par l'État parmi les propriétés sociales des dirigeants de grandes entreprises. Ce particularisme pouvait s'expliquer en partie par le caractère encore très franco-français des grandes entreprises ou par la position prépondérante que l'État occupait dans l'économie du pays. Mais qu'en est-il quelque trente ans plus tard à l'heure de l'économie mondialisée et privatisée ? La part de la France dans le chiffre d'affaires des grandes entreprises cotées au CAC 40 ne cesse de décroître - elle est souvent inférieure à $50 \%$-alors que les privatisations et la libéralisation du crédit ont distendu, pour ne pas dire rompu, les liens de subordination à l'État (Dudouet, Grémont, 2010). Ces transformations de l'environnement des grandes entreprises françaises ont-elles conduit à une modification substantielle du mode de recrutement des dirigeants ? De plus, la massification de l'enseignement supérieur depuis les années 1960 et la multiplication des filières devraient statistiquement favoriser la diversification des parcours scolaires au sein de la génération actuelle. Des éléments de réponse sont apportés par une étude empirique du capital scolaire des quelque 900 dirigeants des entreprises cotées du

1. Institut de recherches interdisciplinaires en sciences sociales (IRISSO) Paris-Dauphine.

2. ISH-Cnrs, Université Lumière Lyon 2.

3. Voir Joly (2007) pour une revue critique de cette littérature. 
CAC 40 à la fin 2007 (cf. encadré méthodologique). Une première constatation s'impose, ces entreprises sont significativement internationalisées : $28 \%$ des dirigeants étaient de nationalité étrangère, dans des proportions, qui varient entre $0 \%$ chez Bouygues et $78 \%$ chez ArcelorMittal. Si le phénomène a beaucoup progressé depuis une vingtaine d'années, la grande majorité des dirigeants des entreprises du CAC 40 (membres des conseils et des comités exécutifs) reste cependant française, plus encore si l'on ne considère que les « patrons » (PDG ou assimilés), qui le sont à $86 \%$ (55 sur 64). Étudier les filières nationales d'accès à ces fonctions apparaît donc toujours pertinent, celles des étrangers reposant a priori sur des facteurs propres aux différents pays concernés.

\section{Méthode et sources}

Par dirigeants, nous entendons, d'une part, l'ensemble des membres des conseils d'administration et de surveillance, y compris les « censeurs » siégeant avec voix consultative, et, d'autre part, les membres des «étatsmajors » opérationnels, généralement rassemblés au sein des « comités exécutifs ». Une sous-population de «patrons » rassemble les présidents de conseil et de directoire, le directeur général stricto sensu au sens de la loi sur les nouvelles régulations économiques de 2001 lorsque le président du conseil d'administration n'assure pas lui-même cette fonction et le principal gérant pour les sociétés en commandite par actions (Lagardère et Michelin). Sur 900 dirigeants (non compris les 45 représentants des salariés dans les conseils tous nationaux) exerçant 1072 fonctions, 627 ont été identifiés comme français (pour quelques-uns par naturalisation, avec une éventuelle double nationalité). La formation supérieure est précisément connue pour $93^{\circ} \%$ d'entre eux. L'exploitation systématique des annuaires papier ou électronique a toujours permis de repérer leur statut éventuel d'ancien élève d'une « très grande école» ou de membre d'un grand corps de l'État (cf. définitions infra).

La dimension patrimoniale continue de jouer à plein, mais pour un nombre extrêmement réduit d'individus. Parmi les membres des conseils, ils ne sont que $12 \%$ à devoir leur position à la détention personnelle ou familiale d'une partie significative du capital d'une entreprise du corpus ou non. Le phénomène est encore plus net dans les états-majors, où les « propriétaires » ne sont que 12 sur 549 (2\%), avec une proportion toutefois nettement plus forte pour les seuls patrons ( 9 sur 64 ). L'immense majorité des dirigeants doit donc sa désignation à d'autres facteurs : ce sont, de manière quasi-exclusive dans les états-majors et de manière dominante 
dans les conseils ${ }^{4}$, des «managers », c'est-à-dire des dirigeants qui ne sont ni des actionnaires influents ni des héritiers. Quels sont les autres facteurs de sélection possibles ? Une première discrimination évidente est celle liée au sexe : les progrès très lents de la parité font que les dirigeants sont à plus de $92 \%$ des hommes; les femmes ne sont qu'un peu mieux implantées dans les conseils (9\%) que dans les états-majors (6\%) grâce aux héritières et aux représentantes des salariés. Pour le reste, on peut trouver deux explications possibles à la sélection opérée. La première, mise en avant par les entreprises elles-mêmes, mettrait l'accent sur les mérites professionnels exceptionnels que ces dirigeants auraient démontrés au cours de leur carrière. La seconde, qui serait celle d'une sociologie critique (Bourdieu, Saint-Martin, 1978 ; Bourdieu, 1989), insisterait au contraire sur le caractère socialement faussé de cette sélection : même s'ils ne sont pas des héritiers du capital économique, ces dirigeants devraient largement leur fonction à la reproduction d'un capital social hérité. Les deux conceptions ne s'opposent d'ailleurs pas nécessairement : les mérites professionnels sont aussi socialement construits dans la mesure où ils reposent largement sur des qualités d'autant plus affirmées qu'elles sont développées depuis l'enfance (Hartmann, 2002 et 2005). Mais leur impact respectif apparait difficilement mesurable dans une enquête empirique : d'une part, les mérites professionnels ne peuvent pas être facilement objectivés et comparés à ceux de populations plus larges ; d'autre part, les données sur les origines sociales, même avec le seul critère réducteur de la profession des parents, ne sont pas disponibles de manière suffisamment complète pour des dirigeants actuels. Par ailleurs, sauf à en rester à un traitement superficiel qui ne mettrait l'accent que sur la présence frappante de quelques grandes familles de l'aristocratie ou de la bourgeoisie des affaires ou qui adopterait une définition très large des « classes dominantes », la « reproduction sociale » ne peut pas tout expliquer : si très rares sont probablement les patrons issus des milieux populaires, nombreux sont ceux, à l'image de Jean-Louis Beffa (SaintGobain), fils d'un ingénieur niçois ou de Gérard Mestrallet (Suez), fils d'un commerçant parisien, qui ont des origines dans une classe moyennesupérieure qui ne suffit pas à les discriminer. Comme l'ont déjà montré des travaux antérieurs (Bauer et Bertin-Mourot, 1987, 1997 ; Maclean, Harvey et Press, 2006 ; Joly, 2008 ; Dudouet et Grémont, 2010), le facteur de l'élitisme scolaire souvent associé au passage par la haute fonction

4. Il y apparaît aussi, parmi les membres des conseils, des personnalités directement issues du monde administratif, politique ou académique. 
publique, avec ces institutions spécifiquement françaises que sont les grandes écoles et les grands corps, apparaît beaucoup plus déterminant. La spectaculaire survivance de ces deux facteurs sera donc successivement décrite dans cet article.

\section{Le poids de l'élitisme scolaire chez les dirigeants français}

Sous réserve des informations lacunaires ( $42 \mathrm{cas}$ ), il ne se trouve que 10 dirigeants français connus pour ne pas avoir pas fait d'études supérieures (post-baccalauréat). Le capital scolaire apparaît donc plutôt élevé. La représentation des universités ou institutions assimilées est en apparence importante avec $42 \%$ des dirigeants connus pour en être diplômés ; mais si l'on ne considère que ceux qui y ont fait leur formation de manière exclusive, la proportion tombe à $14 \%$ (cf. Tableau 1). Les grandes écoles se taillent la part du lion : $84 \%$ des dirigeants français en sont issus, dont $67 \%$ des « très grandes écoles » $(\mathrm{TGE})^{5}$. Les dirigeants propriétaires se distinguent par un niveau moyen de formation plus faible, mais qui reste cependant élevé : ils sont encore $36 \%$ à sortir des TGE, et tous ne sont pas des fondateurs de leur groupe comme Bernard Arnault (X) ou Romain Zaleski (X Mines) ; chez les héritiers, le capital économique n'exclut pas la recherche d'un capital scolaire qui donne un surcroît de légitimité pour s'imposer à la fois au sein de la famille comme de l'entreprise et vis-à-vis des autres actionnaires. Les «liens patrimoniaux » représentent cependant le plus fort taux de «sans formation supérieure » avec $10 \%$, alors que les autres catégories ne dépassent pas $1 \%$ : c'est le cas de grands noms du capitalisme français comme François Pinault, Patrick Ricard ou Martin Bouygues. Ces exceptions concernent toutefois plutôt des dirigeants en fin de carrière. Les nouvelles générations, à l'image de François-Henri Pinault (HEC), sont beaucoup moins susceptibles d'avoir échappé à la massification de l'enseignement supérieur depuis la fin des années 1960.

5. Elles rassemblent ici les écoles les plus sélectives à l'issue des classes préparatoires : Polytechnique (X), Centrale Paris, Mines de Paris, Ponts, les Télécoms et Supélec pour les filières d'ingénieurs ; HEC, l'ESSEC et l'ESCP pour les écoles de commerce et les ENS pour les filières scientifiques et littéraires, ainsi que l'IEP de Paris (Sciences Po) et l'ENA aux concours spécifiques, enfin l'INSEAD comme formation complémentaire. Les autres grandes écoles correspondent aux établissements qui sont aujourd'hui membres de la Conférence des grandes écoles ou qui du moins offrent des formations sélectives semblables. 
Tableau 1 : Distribution unique des dirigeants du CAC 40 par type de formation

\begin{tabular}{|c|c|c|c|c|c|c|}
\hline $\begin{array}{c}\text { Nationalité } \\
\text { type }\end{array}$ & TGE & $\begin{array}{c}\text { Autres } \\
\text { grandes écoles } \\
\text { sans TGE }\end{array}$ & $\begin{array}{c}\text { Université } \\
\text { sans GE }\end{array}$ & $\begin{array}{c}\text { Autres } \\
\text { formations } \\
\text { seules }\end{array}$ & $\begin{array}{c}\text { Pas d'études } \\
\text { supérieures }\end{array}$ & Total \\
\hline Total & 392 & 97 & 79 & 7 & 10 & 585 \\
\hline Patrimonial & 18 & 9 & 7 & 0 & 5 & 39 \\
\hline Autres & 374 & 88 & 72 & 7 & 5 & 546 \\
\hline
\end{tabular}

Parmi les dirigeants dépourvus de liens patrimoniaux, la probabilité d'accéder aux sommets du CAC 40 sans être passé par les grandes écoles est donc faible. Trois écoles (X, ENA et HEC) fournissent à elles seules $46 \%$ des dirigeants français. Les étapes successives de la sélection scolaire que ces établissements couronnent pour aboutir à des promotions annuelles cumulées de moins d'un millier d'élèves à partir de classes d'âge d'environ 800000 personnes conditionnent donc très largement le recrutement des élites économiques françaises. Même si, bien sûr, tous leurs élèves ne peuvent statistiquement devenir dirigeants du CAC 40, les entreprises n'effectuent plus qu'une sélection complémentaire au sein d'un vivier restreint.

\section{La prédominance préservée de Polytechnique dans les filières d'ingénieurs}

Parmi les 247 dirigeants issus des grandes écoles d'ingénieurs, plus de la moitié (126) sont polytechniciens ( $X »)$, ce qui constitue une prééminence spectaculaire pour un établissement qui ne forme dans ces générations que 300-350 élèves parmi plus de 10000 diplômés. L'école est particulièrement bien placée parmi les patrons (19 cas). Elle est représentée dans 34 conseils - quatre d'entre eux (EDF, Axa, SaintGobain et Total) comptant jusqu'à cinq polytechniciens - et dans 30 états-majors - avec un record de 14 X sur 24 membres chez Vinci. Le décalage est d'autant plus grand avec les établissements suivants que certains apparaissent surtout comme écoles d'application de Polytechnique : ils ne sont ainsi que 9 mineurs de Paris sur 47 et 6 Ponts sur 31 à ne pas être également $\mathrm{X}$ (soit à titre du corps ou à titre d'ingénieurs civils). Le décalage avec Centrale Paris (23) en particulier est frappant : alors que les effectifs par promotion sont semblables, cette école qui a pourtant, à la différence de Polytechnique, une vocation exclusive à former des ingénieurs civils, destinés donc aux seules entreprises, est 
près de 6 fois moins implantée parmi les dirigeants du CAC 40. La préférence à peu près systématique accordée depuis plus d'un siècle et demi par les candidats reçus aux deux concours pour l'école militaire apparaît donc pleinement justifiée par ces probabilités très différentes d'accès aux élites. Le maintien de cette prépondérance ancienne de Polytechnique est particulièrement frappante concernant des générations formées pour l'essentiel depuis les années 1960, qui bénéficiaient déjà d'un éventail beaucoup plus étendu de filières de formations supérieures techniques (Joly, 2008). En sens inverse, il est vrai que l'école est devenue dans l'après-guerre surtout une école d'ingénieurs civils qui ne pourvoit plus, malgré le statut qu'elle conserve, que marginalement aux emplois militaires.

Les autres écoles d'ingénieurs sont très largement distancées et n'apparaissent que de manière marginale dans le corpus. Seule l'École spéciale de travaux publics, grâce à la présence au sein du CAC 40 de deux groupes de BTP (Bouygues et Vinci), se distingue modestement (11). Ce résultat est d'autant plus spectaculaire aujourd'hui que toutes ces écoles ont, depuis les années 1960 au moins, des promotions annuelles qui, si elles sont moins nombreuses que celles de Polytechnique, dépassent généralement la centaine d'élèves. Même si ceux-ci ont des parcours scolaires proches, le fait d'avoir « raté la dernière marche » se paie donc cher.

À l'exception des formations médicales présentes à la marge dans le seul groupe pharmaceutique de l'indice, Sanofi-Aventis, les formations universitaires n'apparaissent presque pas au sein des filières scientifiques, de manière exclusive du moins; rares sont d'ailleurs les élèves des grandes écoles d'ingénieurs à avoir complété leur cursus par un doctorat.

\section{L'ascension des filières commerciales dominées par HEC}

L'importance de la filière des grandes écoles commerciales au sein des élites économiques est un phénomène beaucoup plus récent, qui s'est développé surtout à partir des années 1960, lorsque leur recrutement est devenu plus sélectif après un passage obligatoire en classes préparatoires post-baccalauréat (Joly, 2008). Il peut s'interpréter autant comme le reflet d'une importance croissante des enjeux financiers et commerciaux dans la gestion des entreprises que comme une reconfiguration des lieux de formation des élites au profit des établissements 
traditionnellement plus éloignés de l'État. Dans ces filières, on retrouve la même prépondérance de l'établissement choisi de manière privilégiée par les élèves des classes préparatoires reçus à plusieurs concours : HEC - la seule école à ne pas recruter sur liste complémentaire - se détache nettement avec 67 diplômés au sein du CAC 40, contre 26 pour l'ESSEC et 15 pour l'ESCP, alors que les promotions sont de tailles à peu près semblables. Les choix effectués par les meilleurs élèves s'avèrent donc là encore rentables.

Les autres grandes écoles de commerce, en particulier celles de province, sont très largement distancées. Elles forment pourtant plusieurs milliers de diplômés par an dans les générations étudiées. Même l'ESC (devenue EM) Lyon, qui arrive juste derrière les trois grandes dans les choix des candidats aux concours, n'est présente que de manière marginale (3 dirigeants en formation principale), à égalité avec l'ESC Reims, l'ESC Rouen et l'Institut commercial de Nancy.

L'hégémonie des grandes écoles commerciales n'est toutefois pas aussi complète que celle des grandes écoles d'ingénieurs dans la filière technique. L'importance ancienne des facultés de droit dans la formation des élites économiques, notamment dans le secteur financier, n'a pas disparu, d'autant plus que leurs filières de formation se sont diversifiées et professionnalisées avec l'émergence de la gestion comme discipline autonome dans l'après-guerre (création des IAE notamment). L'université Paris Dauphine occupe ainsi une place particulière ( 9 dirigeants dont 3 en association avec une très grande école). Les diplômés de l'université restent cependant relativement peu nombreux au sein des élites du CAC 40, du moins à titre de formation exclusive. Ils sont d'ailleurs surtout représentés dans les fonctions donnant moins souvent accès aux directions générales, comme les directions administratives et juridiques ou celles des ressources humaines.

\section{Le poids du couple "Sciences Po-ENA »}

Si l'ancienne École libre des sciences politiques avait déjà une bonne représentation au sein des élites économiques, notamment bancaires, le phénomène s'est accentué avec sa nationalisation en 1945 et le lien établi avec la nouvelle ENA.

Sciences Po Paris arrive en deuxième position des établissements les plus représentés (113 diplômés en formation initiale) derrière Polytechnique (126). Là encore, l'écart est spectaculaire avec les autres IEP de 
province créés dans l'après-guerre, qui ne sont représentés que marginalement (7 diplômés en tout). Sciences Po Paris pourrait certes, avec sa section économique et financière, être assimilée aux grandes écoles de commerce, mais ce n'est pas la plus fréquentée par les dirigeants du CAC 40 (16 diplômés seulement). La section de service public qui prépare aux concours administratifs l'est beaucoup plus (79). Cette différence s'explique par l'importance des ambitions, concrétisées ou pas, d'intégrer l'ENA.

L'ENA est à l'origine une école qui a une vocation exclusive à la formation des hauts fonctionnaires civils. La démission à la sortie est une pratique qui reste beaucoup plus rare qu'à Polytechnique. L'ENA occupe pourtant une place importante parmi les élites du CAC 40 ; elle se situe à la troisième place des établissements les plus fréquentés (95 anciens élèves), loin devant HEC (64) ou Centrale (23) par exemple, alors que ses effectifs sont plus faibles. Elle est plus représentée dans les conseils (33 sur 40) - avec jusqu'à 5 membres à Saint-Gobain - que dans les états-majors (25, avec cinq absences cumulées dans les deux organes) - et un maximum de 9 membres au Crédit agricole. Ce décalage peut refléter son poids plus grand chez les financiers, mais aussi une possible tendance au déclin dans les générations plus jeunes. Elle est particulièrement bien représentée parmi les patrons (14).

L'intégration à l'ENA ne s'effectue pas à la sortie des classes préparatoires ; chez les dirigeants du corpus, le diplôme bac +3 exigé correspond presque toujours à un passage préalable dans une autre grande école : Sciences Po Paris apparaît de loin comme la voie d'accès privilégiée (64 cas), loin devant HEC (12 dont 4 passés également par Sciences Po Paris), Polytechnique (10), les ENs (6 dont 4 Sciences Po Paris), etc. Ils ne sont que trois énarques à n'avoir été formés qu'à l'université ou dans un IEP de province... L'ENA apparaît donc plutôt comme une composante supplémentaire à l'élitisme scolaire français. Sa position au sommet du système de sélection confirme l'importance des passages par l'État pour l'accès aux élites économiques même privées.

Le poids particulier de Polytechnique, de l'ENA et d'HEC dans le cursus des dirigeants français révèle au moins une étroite symbiose des milieux économiques avec le système français de production des élites. 


\section{La survivance de la relation à l'État à travers les grands corps}

Le CAC 40 rassemble des entreprises exploitées en sociétés par actions à capitaux pour l'essentiel privés ; deux seulement en 2007 (EDF et GDF) peuvent être considérées comme des entreprises publiques stricto sensu dans la mesure où la majorité du capital appartient à l'État, qui est par ailleurs le principal actionnaire minoritaire de trois autres (Air FranceKLM, France Télécom, Renault). Il n'y a donc que dans cinq entreprises où des fonctionnaires en exercice représentent le ministère de tutelle au conseil d'administration et il n'y a que chez EDF, GDF et France Télécom - du fait du statut particulier de l'entreprise qu'elle a conservé depuis sa privatisation - où des fonctionnaires peuvent y faire carrière en détachement. Ailleurs, l'État n'a en principe aucune prise sur la nomination des dirigeants. Pourtant, on observe qu'une part importante des dirigeants français du CAC 40 (28\%) sont d'anciens « serviteurs de l'État » (Dudouet, Grémont, 2007), aussi bien dans les conseils (34\%) que dans les états-majors ( $26 \%)$.

\section{Une filière du pantouflage ${ }^{6}$ qui a survécu aux privatisations}

Les entreprises à participation publique ont certes un taux moyen élevé (41\%) de dirigeants venus de l'État, mais elles sont loin d'être les seules. La présence significative d'anciens hauts fonctionnaires dans d'autres entreprises pourrait s'expliquer par la privatisation relativement récente (depuis 1986) de certaines d'entre elles, comme Saint-Gobain ( $82 \%$ ), BNP-Paribas (45\%), Total $(56 \%)$ ou la Société générale (38\%), ou par l'intégration d'anciennes entreprises publiques, comme l'UAP par Axa (33\%) en 1996 ou le Crédit local de France fusionné, la même année, avec le Crédit communal de Belgique pour former Dexia (62 \%). Toutefois, une partie de ces entreprises n'ont été que très brièvement publiques (entre 1982 et 1986-1987 pour Paribas ou Saint-Gobain) à un moment où beaucoup des dirigeants de 2007 n'étaient pas encore recrutés. De plus, le phénomène touche aussi fortement des entreprises qui n'ont jamais été nationalisées, comme Veolia (59\%), Vinci (50\%) ou Vallourec $(33 \%)$. Essilor est la seule à y échapper totalement. Le phé-

6. Voir dans ce numéro l'article de Luc Rouban. 
nomène, qui n'avait pas commencé avec les nationalisations (Joly, 2008), a donc largement survécu aux privatisations, d'une manière qui ne peut s'expliquer par le simple prolongement des carrières en cours.

Les dirigeants passés par l'État sont aussi particulièrement bien implantés parmi les patrons ( $42 \%$ ) et parmi les multi-administrateurs, ce qui explique que la part des postes qu'ils détiennent (38\%) soit plus importante que leur part dans l'ensemble de la population (28\%). Le phénomène est ainsi beaucoup plus important que le facteur patrimonial dans la dévolution des fonctions dirigeantes ; il obéit d'ailleurs à d'autres logiques que celle de la propriété du capital. Le point commun des entreprises à forte composante étatique pourrait être d'appartenir à des branches d'activités en relation traditionnelle avec un grand corps d'État de contrôle, comme l'inspection des Finances dans les banques ou les Ponts et Chaussées dans le BTP (Vinci), dont les membres tendent souvent à prolonger leur carrière dans les entreprises qu'ils surveillaient en tant que fonctionnaires, et/ou des branches fortement dépendantes des commandes publiques (Veolia, Vinci, Alsthom). Mais le phénomène devrait avoir décliné, d'une part, avec la déréglementation marquée depuis les années 1980 qui a, notamment dans les banques, restreint le pouvoir des administrations de contrôle sur les entreprises et, d'autre part, avec l'internationalisation croissante des activités qui a diminué la part des commandes publiques nationales dans le chiffre d'affaires global. Il ne peut ainsi pas expliquer un pourcentage très élevé, comme celui de Saint-Gobain, aux activités très internationalisées et de plus en plus tournées vers les marchés privés des matériaux de construction, qui repose à l'évidence sur une tradition plus ancrée de recrutement des élites à la française. La pratique du « pantouflage » est aussi en principe encadrée par une réglementation plus stricte, avec l'existence d'une commission de contrôle, même si celle-ci a récemment montré les limites de ses prérogatives.

\section{L'importance de l'appartenance aux grands corps}

La majorité de ces dirigeants «d'État» sont issus de filières très spécifiques. Ils sont en effet 123 sur 178 (69\%) à être issus de l'un des six «grands corps», recrutés dans la «botte» de l'École polytechnique - Mines (33), Ponts (23), Télécoms (11) - ou de l'ena Inspection des Finances (37), Conseil d'État (6), Cour des comptes (13). Ils représentent également $65 \%$ des patrons « d'État» (6 inspection des Finances, 4 Mines, 3 Ponts et 2 Télécoms). On retrouve là le processus 
ultime de production des élites à la française. Si le passage par les grandes écoles est un facteur déterminant pour accéder à la direction des grandes entreprises, il est tout aussi important d'en sortir parmi les mieux classés. Ainsi, c'est la fraction supérieure des polytechniciens, ceux qui intègrent les grands corps techniques de l'État, qui s'en sortent le mieux, non la majorité des démissionnaires qui rejoignent le privé dès la sortie. Le fait que les $\mathrm{X}$ Mines soient ainsi plus nombreux parmi les dirigeants du CAC 40 malgré leur corps plus restreint (une dizaine de places par an à la sortie de l'X pour les générations étudiées) que celui des X Ponts (une trentaine) est à mettre en relation avec le choix à peu près systématique de cette affectation par les meilleurs élèves qui en ont la possibilité. Parmi les énarques, si les démissions à la sortie sont beaucoup plus rares - ils ne sont que trois parmi les dirigeants du CAC 40 à n'avoir pas servi l'État -, c'est également la minorité qui intègre les grands corps de contrôle, et en particulier les inspecteurs des Finances, qui réussissent le mieux.

Il faut toutefois signaler une récente tendance au reflux des deux grands corps. L'inspection des Finances, si elle reste très bien implantée dans le secteur financier, a plutôt décliné dans les autres secteurs, par rapport à la fin des années 1990 où le corps comptait plusieurs PDG dans l'industrie et les services. De même, le corps des Mines apparaît en léger déclin, notamment parce que beaucoup des entreprises métallurgiques ou chimiques qui constituaient ses bastions, comme Usinor-Sacilor, Pechiney ou Kuhlmann, ont disparu et qu'il n'a pas réussi à s'implanter de la même manière dans de nouvelles branches. L'avenir dira si ces évolutions sont conjoncturelles ou durables.

\section{L'importance du passage par les cabinets ministériels}

La grande majorité des dirigeants « d'État » ont quitté le service public avant 40 ans, donc avant l'accès, pourtant très précoce en France pour les membres des grands corps, au sommet de la hiérarchie administrative (direction d'administration centrale). La filière propre au secteur nationalisé des grands directeurs ministériels (du Trésor, des Mines, etc.) pantouflant à 45-50 ans dans des entreprises dont ils avaient la tutelle a décliné avec les privatisations. En revanche, un facteur important ( 99 sur 178, soit $56 \%$ ) apparaît être le passage dans un cabinet ministériel ; celui-ci a lieu souvent dès les premières années de la carrière, dans des fonctions de conseiller plus que de direction et dans des ministères économiquement 
stratégiques comme l'Économie et les Finances, l'Industrie ou l'Équipement, et il précède souvent immédiatement le pantouflage. Les relations avec les milieux d'affaires acquises dans ces fonctions, ou inversement la capacité à faire valoir des entrées dans le monde politique, apparaissent essentielles pour la carrière future. L'orientation politique des cabinets ne semble pas, pour ces générations affectées depuis vingt-cinq ans par des alternances successives, être déterminante. Ils sont presque aussi nombreux à être passés par des cabinets de gauche (44) que de droite (53). C'est justement souvent après une alternance que ceux engagés en faveur de la majorité précédente sont amenés à pantoufler, faute de se voir offrir des fonctions intéressantes par la nouvelle équipe. Les entreprises semblent les accueillir indifféremment, les ressources accumulées dans les fonctions exercées étant probablement plus déterminantes que l'orientation partisane; recruter dans les deux camps permet par ailleurs aux firmes d'y entretenir des réseaux utiles dans chacun d'entre eux en cas de nouvelle alternance.

Même si quelques évolutions ont pu être soulignées, les résultats d'ensemble traduisent une étonnante permanence du mode de production des dirigeants des grandes entreprises françaises. Il reste à savoir si cette situation ne constitue qu' une survivance liée au prolongement de carrières commencées dans un autre contexte ou si elle est appelée à se pérenniser. On peut cependant avancer que cette survivance n'est pas vraiment une surprise dès lors que l'on considère les élites et leurs modes de reproduction pour elles-mêmes et non pour les fonctions qu'elles sont censées occuper. Le maintien des grandes écoles de la République dans la production des élites économiques privées n'est incongru que pour une vision par trop wébérienne de la société qui voudrait que l'organisation légale rationnelle de l'éducation forme à des fonctions (hauts fonctionnaires, managers) avant de produire des statuts (dirigeants). Ainsi que le soulignait déjà Bourdieu, le système scolaire produit d'abord une hiérarchie sociale qui conditionne l'accès différencié au stock de ressources disponibles. La permanence des grandes écoles dans un monde économique privatisé et ouvert sur l'international semble montrer simplement deux choses: 1) qu'il n'existerait pas encore de véritable mode transnational de reproduction des élites économiques 2) que le système français de production des élites, longtemps étroitement associé à l'État, parviendrait pour l'heure à s'affranchir de son déclin.

Francois_Xavier.dudouet@dauphine.fr Herve.joly@ish-lyon.cnrs.fr 


\section{Références bibliographiques}

Bauer M., Bertin-Mourot B. (1987), Les 200. Comment devient-on un grand patron? Paris, Le Seuil.

BAUER M., BERTIN-Mourot B. (1997), Radiographie des grands patrons français. Les conditions d'accès au pouvoir, Paris, L'Harmattan.

Bourdieu P. (1989), La Noblesse d'État. Grandes écoles et esprit de corps, Paris, Minuit.

Bourdieu P., de Saint-Martin M. (1978), "Le Patronat », Actes de la recherche en sciences sociales, $\mathrm{n}^{\circ}$ 20/21, pp. 3-82.

Dudouet F.-X., GrÉmont É. (2007), « Les grands patrons et l'État en France, 1981-2007 », Sociétés Contemporaines, №68, pp. 105-131.

Dudouet F.-X, GrÉMONT É. (2010), Les grands patrons en France. Du capitalisme d'État à la financiarisation, Paris, Lignes de Repères.

Hartmann M. (2002), Der Mythos von den Leistungseliten. Spitzenkarrieren und soziale Herkunft in Wirtschaft, Politik, Justiz und Wissenschaft, Francfort/M., Campus.

HARTMANN M. (2005), "Le recrutement des dirigeants des grandes entreprises en Allemagne. Une sélection sociale en l'absence d'institutions de formation des élites ", in Joly H.(dir.). Formation des élites en France et en Allemagne, Cergy-Pontoise, CIRAC, pp. 83-100.

Joly H. (2007), « Le recrutement du patronat », Sociétés Contemporaines, $\mathrm{n}^{\circ} 68$, pp. 133-154.

Joly H. (2008), Diriger une grande entreprise française au $x x^{e}$ siècle : modes de gouvernance, trajectoires et recrutement, mémoire inédit d'habilitation à diriger des recherches, Paris, EHESS.

Maclean M., Harvey C., Press J. (2006), Business Elites and Corporate Governance in France and the UK, Basingstoke, Palgrave Macmillan. 